\title{
Radiative heat transfer in a model gas turbine combustor
}

\author{
M. C. Paul ${ }^{1} \&$ W. P. Jones ${ }^{2}$ \\ ${ }^{1}$ Department of Mechanical Engineering, University of Glasgow, UK \\ ${ }^{2}$ Department of Mechanical Engineering, Imperial College London, UK
}

\begin{abstract}
In this paper we have carried out a three-dimensional numerical study to investigate the radiative heat transfer in a model gas turbine combustor, representative of the Rolls-Royce Tay combustor. The Discrete Ordinate Method (DOM/Sn) in general body-fitted co-ordinate system is applied to solve the filtered Radiative Transfer Equation (RTE) for the radiation modelling and this has been combined with a Large Eddy Simulation (LES) of the flow, temperature and composition fields within the combustion chamber. The radiation considered in this work is due only to the hot combustion gases notably carbon dioxide $\left(\mathrm{CO}_{2}\right)$ and water vapour $\left(\mathrm{H}_{2} \mathrm{O}\right)$ also known as non-luminous radiation. The instantaneous results of the radiation properties such as the incident radiation and the radiative energy source or sink as the divergence of the radiative heat fluxes are computed inside the combustion chamber and presented graphically.

Keywords: discrete ordinates method, large eddy simulation, radiative heat transfer, turbulent combustion.
\end{abstract}

\section{Introduction}

A large part of the total heat transfer in a combustion chamber, whether it is a gas turbine engine, a car engine or a furnace, occurs by radiation from the flame. This radiation has two components: (i) the 'non-luminous', which emanates from the combustion gases notably carbon dioxide $\left(\mathrm{CO}_{2}\right)$ and water vapour $\left(\mathrm{H}_{2} \mathrm{O}\right)$, and (ii) the 'luminous', which is mainly due to the soot formed in the flame. The prediction of wall temperatures is an important aspect in the design of practical engine combustors and this clearly requires that the radiative heat fluxes be predicted accurately. An inability to predict the wall temperatures may lead to an exces- 


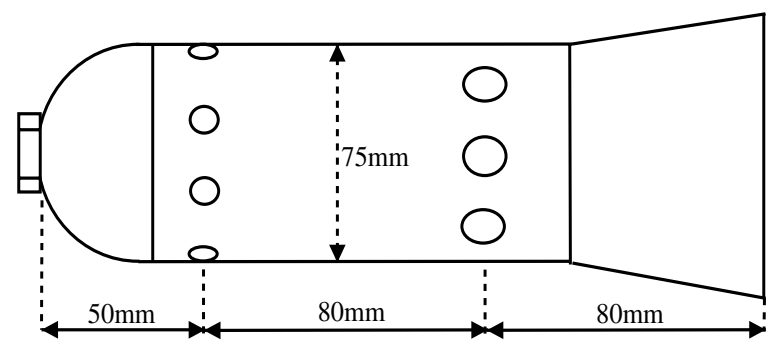

Figure 1: The feature of a model Tay gas turbine combustor.

sive amount of the combustor airflow being used for cooling the liner wall and this is likely to lead to a reduced combustion efficiency and an increased emission of the pollutants such as carbon monoxide $(\mathrm{CO}), N \mathrm{O}_{x}$ formations and unburned hydrocarbons $(U H C)$. In addition, excessive combustor wall temperatures have a deleterious impact on combustor 'life'.

The Discrete Ordinates Method (DOM) was first proposed by Chandrasekhar [1] in his work on one-dimensional stellar and atmospheric radiation. Subsequently Carlson and Lathrop [2] developed the DOM for multi-dimensional radiation problems employing the finite volume approach. More recently, the DOM has been widely used on various different problems [3-5] where the major emphasis has been on solving the Radiative Transfer Equation (RTE), which is the steady state representation of the radiative transfer. The radiative transfer in high-temperature combustion devices requires a simultaneous solution of the RTE and the governing flow equations such as Navier-Stokes, enthalpy and species concentration conservation equations, etc, [6-8].

With respect to combining the RTE with the LES, to the authors' knowledge only a small amount of work has been done to date. Desjardin and Frankel [7] studied soot formation in the near field of a strongly radiating turbulent jet flame involving LES and a simplified two-dimensional treatment of radiation involving gray and non-scattering medium was considered. Recently, Jones and Paul [8] have investigated the radiative heat transfer in a three-dimensional model of a gas turbine combustor, where the $S_{4}$ approximation of the DOM was applied in conjunction with LES. In this paper we have extended the DOM including its various lower and higher order approaches, ie $S_{2}, S_{6}, S_{8}$, and applied into the model Tay gas turbine combustion chamber for further investigation of the radiative heat transfer and to see how the radiation results are affected with the various order of approximations of the DOM. To the best of our knowledge, there have been no other attempts that combine the three-dimensional DOM with LES for computing the turbulent flame radiation in a gas turbine combustor. 


\section{Governing equations}

The main features of the model gas turbine combustor are shown in fig. 1, which is representative of the Rolls-Royce Tay gas turbine [9]. The combustor walls are made of transply, a laminated porous material. The geometry of the combustor includes a relatively small swirler at the head of the combustor in the centre of which the fuel injector is located and a hemispherical head section attached to a circular barrel of $75 \mathrm{~mm}$ diameter. This barrel contains a set of six primary ports/holes of $10 \mathrm{~mm}$ diameter each at the front and another set of six dilution ports/holes of $20 \mathrm{~mm}$ diameter each at $80 \mathrm{~mm}$ downstream of the first set. A circular-torectangular nozzle is attached to the end of the barrel. High purity gaseous fuel comprising over $95 \%$ propane $\left(\mathrm{C}_{3} \mathrm{H}_{8}\right)$ was injected into the combustion chamber through the centre of the swirler.

The equations of motion in LES are obtained after applying a spatial filter [10] and a density weighted Favre filter [11] to the continuity, the Navier-Stokes, and the mixture fraction equations. In DOM the discrete ordinates representation of the radiative transfer equation is filtered using the spatial filter. Finally these equations take the following forms [8]:

$$
\begin{gathered}
\frac{\partial \bar{\rho}}{\partial t}+\frac{\partial \bar{\rho} \tilde{u}_{j}}{\partial x_{j}}=0 \\
\frac{\partial \bar{\rho} \tilde{u}_{i}}{\partial t}+\frac{\partial \bar{\rho} \tilde{u}_{i} \tilde{u}_{j}}{\partial x_{j}}=-\frac{\partial \bar{p}}{\partial x_{i}}+\frac{\partial}{\partial x_{j}}\left(2 \mu \bar{S}_{i j}-\frac{2}{3} \mu \bar{S}_{k k} \delta_{i j}\right)-\frac{\partial \tau_{i j}}{\partial x_{j}}, \\
\frac{\partial \bar{\rho} \tilde{f}}{\partial t}+\frac{\partial \bar{\rho} \tilde{u}_{j} \tilde{f}}{\partial x_{j}}=\frac{\partial}{\partial x_{j}}\left(\frac{\mu}{S c} \frac{\partial \bar{f}}{\partial x_{j}}\right)-\frac{\partial J_{j}}{\partial x_{j}}, \\
\alpha_{m} \frac{\partial \bar{I}_{m}}{\partial x}+\beta_{m} \frac{\partial \bar{I}_{m}}{\partial y}+\gamma_{m} \frac{\partial \bar{I}_{m}}{\partial z}+\overline{\kappa I_{m}}=\overline{\kappa I_{b}} .
\end{gathered}
$$

where $\rho$ is the mixture density, $t$ is the time, $x_{j}=(x, y, z)$ is the coordinate vector, $u_{j}$ is the velocity vector, $p$ is the dynamic pressure, $\mu$ is the coefficient of viscosity, $S_{i j}$ is the strain rate defined as $S_{i j}=\frac{1}{2}\left(\frac{\partial u_{i}}{\partial x_{j}}+\frac{\partial u_{j}}{\partial x_{i}}\right), \delta_{i j}$ is the Kronecker delta, $f$ is the mixture function and $S c$ is the Schmidt number.

In eqn (4), $I_{m}$ represents the radiative intensity along the angular direction, where $m=1,2, \ldots, M$ (see $[8,10]$ for a detailed angular representation), thus the equation represents a set of $M$ different directional radiative intensities from a computational grid node. The terms $\alpha_{m}, \beta_{m}$ and $\gamma_{m}$ in eqn (4) represent the direction cosines of an angular direction along the coordinates and $I_{b}$ is the blackbody intensity at the temperature of the medium which is defined as $\frac{\sigma \tilde{T}^{4}}{\pi}$ where $\sigma$ is the Stefan-Boltzmann constant and $\tilde{T}$ is the flame temperature. $\kappa$ is the absorption coefficient, and for a non-luminous radiation, which is considered in this work, it is a function of the mole fractions of $\mathrm{H}_{2} \mathrm{O}$ and $\mathrm{CO}_{2}$ (see [8]). For the radiation modelling we have also assumed that the enclosure contains an absorbing-emitting, non-scattering and radiatively gray medium. 
In eqns (2)-(3) the sub-grid-scale stresses, $\tau_{i j}$, and the sub-grid-scale scalar fluxes, $J_{j}$, are modelled using the standard Smagorinsky model [13] and a gradient model [14] respectively. For the unknown terms, $\overline{\kappa I_{m}}$ and $\overline{\kappa I_{b}}$, in eqn (4), which are the nonlinear correlations between turbulence and radiation, we have ignored the sub-grid-scale turbulence interaction with radiation. A future study is required to incorporate these interactions and to investigate their effects in the radiative heat transfer predictions in a turbulent flame.

The detailed boundary conditions for solving the filtered eqns (1)-(3) are presented in [15], and while for the filtered RTE (4) these are given in [8] and will not be repeated here.

\section{Numerical procedures}

The filtered equations (1)-(4) are rewritten in general boundary/body fitted coordinates system using the approach introduced by Thompson [16], where the governing differential equations in the Cartesian coordinates are transformed into the curvilinear coordinates system. The details of the numerical procedures in the LES approach to solve eqns (1)-(3) have already been presented in [15, 17] and will not be repeated. The numerical procedures in DOM to solve the RTE (4) can be found in Jones and Paul [8]. Also a benchmark problem was considered in [8] to assess accuracy of the numerical results of the DOM in a general body fitted co-ordinates system, and a very good agreement was obtained compared with the results available in literature, for further details the readers are referred to [8].

\section{Results and discussion}

In the present computation we employed a total of about $10^{5}$ control volumes with $40 \times 60 \times 40$ grid nodes in the $x, y$ and $z$ directions respectively. The time step in the simulation was chosen to be $d t=5.53 \times 10^{-7}$ ( sec) based on the consideration of the maximum Courant number which never exceeded 0.1 throughout the computations. Overall the LES code is second order accurate in both space and time domains and for the DOM we used the scheme described in [8]. The results presented in figs. $2-4$ are at $6.5 \times 10^{5}$ time steps, which is at the real clock time of $t \approx 0.036 \mathrm{sec}$.

Instantaneous results of the flame temperatures, absorption coefficient and the total radiative intensity, $\bar{I}=\sum_{m=1}^{M} \bar{I}_{m}$, at various horizontal locations of the combustion chamber are presented in fig. 2. The radiation results shown in this figure are obtained applying the highest order approximation of the DOM, $S_{8}$, and the emissivity of walls was kept at 0.5 . We note that the results of temperature and absorption coefficient are obtained initially without considering any radiation effects. These results are then feed into the radiation solver, DOM, to solve the RTE (4). These are required; as the sources of radiation, e.g. the black body intensity $\left(\bar{I}_{b}\right)$, depend on the flame temperature; and the absorption coefficient, $\bar{\kappa}$, which is considered to be a function of the mole fractions of $\mathrm{H}_{2} \mathrm{O}$ and $\mathrm{CO}_{2}$. 
(a)
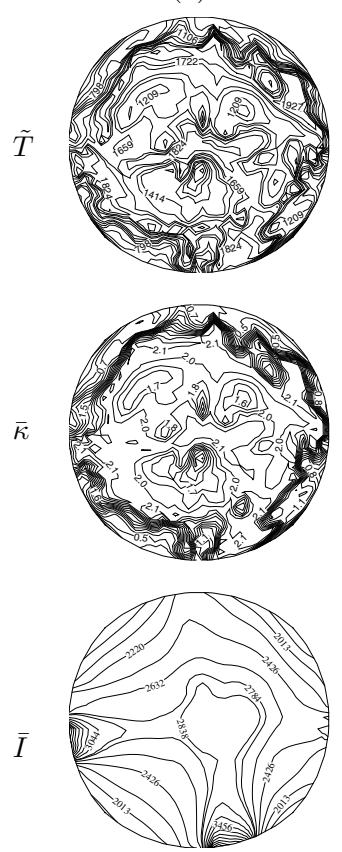

(b)
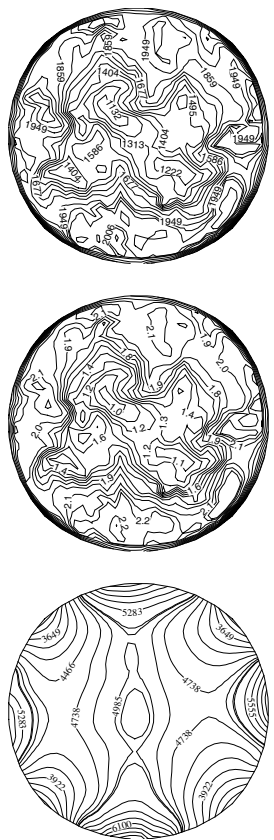

(c)
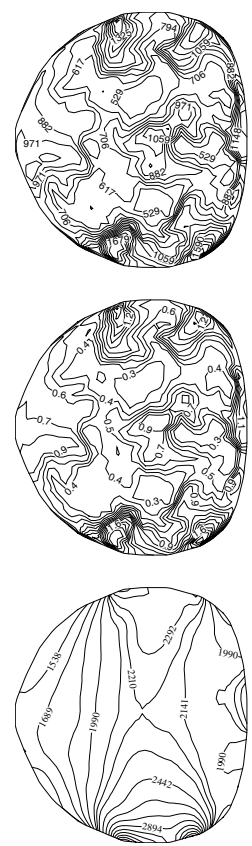

Figure 2: Instantaneous contour plots of $\tilde{T}(K), \bar{\kappa}\left(\mathrm{cm}^{-1}\right), \bar{I}\left(k W m^{-2}\right)$ at (a) $y=20 \mathrm{~mm}$, (b) $y=95 \mathrm{~mm}$ and (c) $y=165 \mathrm{~mm}$.
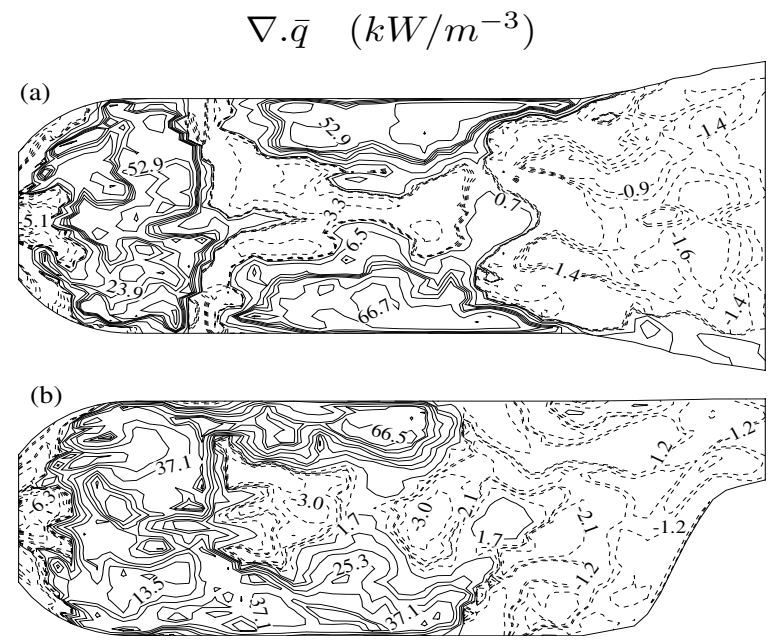

Figure 3: Contours of the divergence of the radiative heat fluxes on (a) the midhorizontal and (b) the mid-vertical planes of the combustor. 

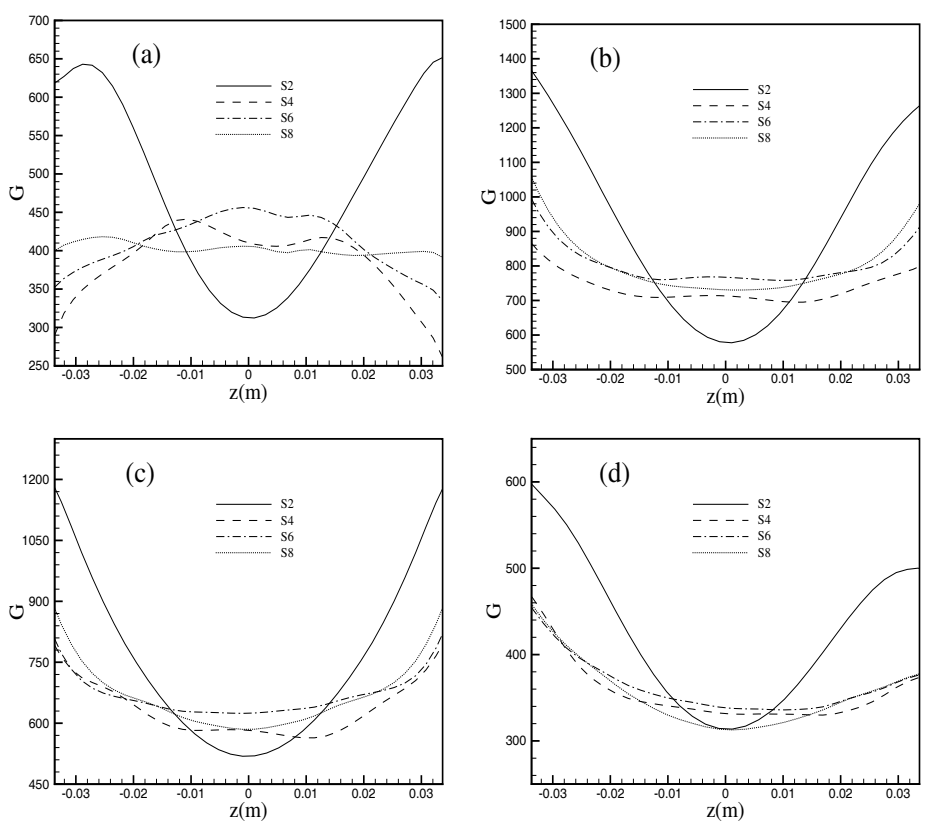

Figure 4: Radial profiles of the incident radiation, $G\left(k W m^{-2}\right)$, at (a) $y=20 \mathrm{~mm}$, (b) $y=95 \mathrm{~mm}$, (c) $y=130 \mathrm{~mm}$ and (d) $y=165 \mathrm{~mm}$ on the midhorizontal plane of the combustor.

A good agreement was achieved when di Mare et al. [15] compared these computational results of $\tilde{T}, \tilde{Y}_{\mathrm{H}_{2} \mathrm{O}}$ and $\tilde{Y}_{\mathrm{CO}_{2}}$ against experimental measurements of Bicen et al. [9], which will not be repeated here. In this figure we can also see that the total radiative intensity attains a maximum at the region where both the temperature and absorption coefficient are maximum. Thus it provides clear evidence that the medium is highly dominated by the hot $\mathrm{H}_{2} \mathrm{O}$ and $\mathrm{CO}_{2}$ gases. The radiative heat flux distributions also become maximum at the position where the radiative intensities are also maximum.

Fig. 3 shows the net loss or gain of the energy due to the radiation as a divergence of the radiative heat fluxes, $\nabla \cdot \overline{\mathbf{q}}$, which have been calculated using the following relation

$$
\nabla \cdot \overline{\mathbf{q}}=4 \pi \bar{\kappa} \bar{I}_{b}-\int_{4 \pi} \bar{\kappa} \bar{I} d \Omega .
$$

In this figure, the dashed lines represent the negative contours. The results obtained using $S_{8}$ version of the DOM and the wall emissivity remains the same, ie $\epsilon_{w}=$ 0.5 .

In eqn (5), the first term on the right hand side represents the emitted/outgoing radiation from a computational control volume, while the second term represents 
the total incident radiation into that control volume. Thus, $\nabla \cdot \overline{\mathbf{q}}$ gives the rate of the generation of energy by radiation and this must be coupled in the overall energy conservation, which turns to be an improved temperature prediction inside the combustion chamber. Further work is required to investigate this effects on the flame temperature and combustion species. However this would require a substantial increase in computer resources as, at every time-step, the radiation intensities would have to be calculated by the radiation solver for updating the radiative heat fluxes and finally obtaining a new temperature for the next time step.

In fig. 4 we show some results of the incident radiation, $G$, at various radial positions on the horizontal plane of the gas turbine combustor. To judge the accuracy of the various orders of DOM $\left(S_{n}\right)$ in the present work, the results of incident radiation are plotted for $S_{2}, S_{4}, S_{6}$ and $S_{8}$. The wall emissivity is still remain unchanged here. It is also interesting to mention here that the incident radiation is an important radiation property related to the radiative energy density, by which the total radiation energy is stored in each computational node, so each frame in this figure shows a distinct variation of the energy storage inside the combustion chamber. Prediction of the incident radiation is also an essential task which allows us the radiative energy transfer to be coupled with the global energy conservation (for example, see eqn. (5)). The incident radiation is calculated using the relation,

$$
G=\int_{4 \pi} \bar{I} d \Omega \approx \sum_{m=1}^{M} \omega_{m} \bar{I}_{m} .
$$

In fig. 4(a)-(d) we can see how the results obtained using the most lower order approximation, $S_{2}$, of the DOM diverts from those with the higher orders. But the higher order $S_{n}\left(S_{4}, S_{6}\right.$ and $\left.S_{8}\right)$ results appear to converge together at a level of $G$. It is worth to mention here that the DOM with $S_{2}$ approximation was also tested considering a relatively smaller wall emissivity, $\epsilon_{w}=0.1$, and a poor convergence rate was achieved in the radiation solver due to the very oscillatory nature of the radiative intensity solutions.

In fig. 4(a), $G$ is calculated in a position of the combustor head and it is found to be lower compared with those in other fames. This is expected as the radiative intensity, absorption-emission rate and flame temperature in the combustor head region are predicted lower (see fig. 2). However, in the combustor barrel (at $y=95 \mathrm{~mm}$, fig. 4(b)), $G$ is predicted to be the highest, because this part of the combustor houses the extremely hot gases. At the dilution ports (at $y=130 \mathrm{~mm}$, fig. 4(c)) the effect of the large amount of cooling air on the prediction of the incident radiation is clear, and at the downstream (at $y=165 \mathrm{~mm}$, fig. 4(d)) $G$ decreases again because of the effect of the cooling air through the dilution ports and no combustion occurs downstream of these ports.

\section{Conclusions}

The $S_{n}$ approximation of the discrete ordinate method has been implemented to investigate the radiative heat transfer inside a model gas turbine combustor. The 
DOM has been combined with a Large Eddy Simulation of the flow, temperature and composition fields within the combustion chamber. A gray-gas and nonscattering approximation to the RTE has been assumed, and the absorption coefficient for both $\mathrm{H}_{2} \mathrm{O}$ and $\mathrm{CO}_{2}$ gases is calculated.

The instantaneous results of the radiative heat fluxes, incident radiation, and divergence of radiative heat fluxes have been calculated. A coupling of this radiative heat gain/loss is likely to yield accurately predicted wall temperature and this will aid combustor design by allowing an optimum amount of air to be used for wall cooling. The beneficial effects will be a reduction in the emission of pollutant gases by maximising the combustion efficiency and to allow a longer liner life.

The present study excludes the effects of the soot on radiative heat transfer. Soot is likely to enhance the radiation field and ultimately the coupling of soot formation and consumption to heat radiation is an important requirement. The soot concentrations are required and this in turn requires the solution of the appropriate conservation equations for the soot properties. Research is currently underway on this.

\section{References}

[1] Chandrasekhar, S., Radiative Transfer. Dover Publications, 1960.

[2] Carlson, B.G. \& Lathrop, K.D., Transport theory-the method of discrete ordinates. Computing Methods in Reactor Physics, Gordon and Breach, New York, pp. 165-266, 1968.

[3] Jamaluddin, A.S. \& Smith, P.J., Predicting radiative transfer in rectangular enclosures using the discrete ordinates method. Comb Sci and Tech, 59, pp. 321-340, 1988.

[4] Liu, J., Shang, H.M., Chen, Y.S. \& Wang, T.S., Prediction of radiative transfer in general body-fitted coordinates. Num Heat Tran Part B, 31, pp. 423439, 1997.

[5] Kayakol, N., Selcuk, N., Campbell, I. \& Gulder, O.L., Performance of discrete ordinates method in a gas turbine combustor simulator. Exp Thermal and Fluid Sci, 21, pp. 134-141, 2000.

[6] Kaplan, C.R., Baek, S.W., Oran, E.S. \& Ellzey, J.L., Dynamics of a strongly radiating unsteady ethylene jet diffusion flame. Combustion and Flame, 96, pp. 1-21, 1994.

[7] Desjardin, P.E. \& Frankel, S.H., Two-dimensional large eddy simulation of soot formation in the near-field of a strongly radiating nonpremixed acetylene-air turbulent jet flame. Combustion and Flame, 119, pp. 121-132, 1999.

[8] Jones, W.P. \& Paul, M.C., Combination of dom with les in a gas turbine combustor. Int J Eng Sci, 43, pp. 379-397, 2005.

[9] Bicen, A.F., Tse, D.G.N. \& Whitelaw, J.H., Combustion characteristics of a model can-type combustor. Tech. rep. fs/87/28, Imperial College London, 1987. 
[10] Leonard, A., Energy cascade in large-eddy simulations of turbulent fluid flows. Advances in Geophysics, 18A, pp. 237-248, 1974.

[11] Favre, A., Statistical equations of turbulent cases in problems of hydrodynamics and continuum mechanics. Technical report, Society of Industrial and Applied Mathematics, Philadelphia, 1969.

[12] Modest, M.F., Radiative heat transfer, second edition. Academic Press, 2003.

[13] Smagorinsky, J., General circulation experiments with the primitive equations. i. the basic experiment. Monthly Weather Review, 91, pp. 99-164, 1963.

[14] Schmidt, H. \& Schumann, U., Coherent structure of the convective boundary layer derived from large eddy simulation. J Fluid Mech, 200, pp. 511-562, 1989.

[15] di Mare, F., Jones, W.P. \& Menzies, K., Large eddy simulation of a model gas turbine combustor. Combustion and Flame, 137, pp. 278-294, 2004.

[16] Thompson, J.F., Thames, F. \& Mastin, C., Automatic numerical generation of body-fitted curvilinear coordinates system for field containing any number of arbitrary two-dimensional bodies. J Comp Phys, 15, pp. 299-319, 1974.

[17] Branley, N. \& Jones, W.P., Large eddy simulation of a turbulent nonpremixed flame. Combustion and Flame, 127, pp. 1913-1934, 2001. 